\title{
Title: The receptor kinase FER is a RALF-regulated scaffold controlling plant immune signaling
}

Authors: Martin Stegmann ${ }^{1}$, Jacqueline Monaghan ${ }^{1 \S}$, Elwira Smakowska-Luzan ${ }^{2}$, Hanna Rovenich $^{1 \pi}$, Anita Lehner ${ }^{3}$, Nicholas Holton $^{1}$, Youssef Belkhadir ${ }^{2}$, Cyril Zipfel $^{1 *}$

\author{
Affiliations: \\ ${ }^{1}$ The Sainsbury Laboratory, Norwich Research Park, Norwich, NR4 7UH, UK. \\ ${ }^{2}$ Gregor Mendel Institute (GMI), Austrian Academy of Sciences, Vienna Biocenter (VBC), \\ Vienna, 1030, Austria. \\ ${ }^{3}$ Protein Technologies Facility, Vienna Biocenter Core Facilities (VBCF), Vienna, Austria. \\ *Corresponding author. E-mail: cyril.zipfel@tsl.ac.uk \\ ${ }^{\S}$ Present address: Biology Department, Queen's University, Kingston, ON, K7L 3N6, Canada. \\ IPresent address: Laboratory of Phytopathology, Wageningen University, 6708 PB Wageningen, \\ The Netherlands.
}

\begin{abstract}
In plants, perception of invading pathogens involves cell-surface immune receptor kinases. Here, we report that the Arabidopsis SITE-1 PROTEASE (S1P) cleaves endogenous RAPID ALKALINIZATION FACTOR (RALF) propeptides to inhibit plant immunity. This inhibition is mediated by the malectin-like receptor kinase FERONIA (FER), which otherwise facilitates the ligand-induced complex formation of the immune receptor kinases EF-TU RECEPTOR (EFR) and FLAGELLIN-SENSING 2 (FLS2) with their co-receptor BRASSINOSTEROID INSENSITIVE 1-ASSOCIATED KINASE 1 (BAK1) to initiate immune signaling. We show that FER acts as a RALF-regulated scaffold modulating receptor kinase complex assembly. A similar scaffolding mechanism may underlie RALF function in other signalling pathways.
\end{abstract}

One Sentence Summary: A broadly accessible receptor perceives antagonistic endogenous peptides to regulate the formation of plant immune receptor complexes.

Main Text: Plant immune pattern recognition receptors (PRRs) are often receptor kinases (1). The Arabidopsis thaliana (hereafter Arabidopsis) receptor kinases FLS2 and EFR bind bacterial flagellin (or the epitope flg22) and EF-Tu (or the epitopes elf18/elf24), respectively, and form ligand-induced complexes with their co-receptor BAK1 (1). 
To decipher the negative regulation of plant PRR-mediated immune signaling, we screened for modifier of bakl-5 (mob) mutants that regain immune responses in the immunodeficient mutant background bakl-5 (2). Here, we report the characterization of MOB6.

bakl-5 is impaired in the production of reactive oxygen species (ROS) upon flg22 or elf18 treatment (3). We identified the recessive bakl-5 mob6 mutant based on restoration of this response (fig. S1A). To map the mob6 locus, we sequenced bulked F2 segregants from a backcross between bakl-5 mob6 and bakl-5 combined with phenotypic and genetic analysis of an F2 population from a cross between bakl-5 mob6 and Col-0 (Supplement). We found a homozygous missense mutation (P612S) in At5g19660 encoding the subtilase S1P/SBT6.1 (fig. $\mathrm{S} 1 \mathrm{~B}, \mathrm{C})$. Allelism tests and trans-complementation assays confirmed that the mob6 phenotype is caused by a mutation in $S 1 P$ (fig. S1D,E). We thus renamed mob6 as $s 1 p-6$.

We characterised the effect of mutant $S 1 P$ alleles on immune signaling. slp-3 and $s 1 p-6$ single mutants produced more ROS in response to elf18, flg22 and chitin (fig. S1D,E, fig. S2A, fig. S3), and exhibited increased expression of the immune marker genes FRK1 and PHI1 upon elf18 treatment (fig. S2B). Also, s1p-3 and $s 1 p-6$ mutants were more resistant to the hypovirulent bacterium Pseudomonas syringae pv. tomato (Pto) DC3000 COR- (fig. S2C). Thus, S1P is a negative regulator of immunity.

Similar to its human ortholog, the Arabidopsis subtilase S1P processes substrates with the canonical cleavage site RxxL/RxLx (4). The endogenous peptide RAPID ALKALINISATION FACTOR 23 (RALF23; At3g16570) - an established S1P substrate in Arabidopsis (5) - is a major hub in a flg22-regulated transcriptional network (6). Consistent with a potential role of RALF23 in immunity, elf18 treatment or inoculation with the type III-deficient strain Pto DC3000 $\mathrm{hrCC}^{-}$rapidly increased the processing of the propeptide PRORALF23 (fig. S4A,B), in a S1P-dependent manner (fig. S4C).

RALF23 treatment led to a dose-dependent inhibition of elf18 ROS (IC $50 \sim 200 \mathrm{nM}$; fig. 1A, fig. S5) and induced-resistance to Pto DC3000 (fig. 1B). Furthermore, RALF23 overexpression (7) inhibited elf18-triggered ROS and increased susceptibility to Pto DC3000 COR (fig. 1C,D) and to the fungus Plectosphaerella cucumerina (7). Conversely, loss of RALF23 (fig. S6A) led to increased elf 18-triggered ROS (fig. 1E) and resistance to Pto DC3000 COR (fig. 1F). RALF23 similarly inhibits flg22-triggered ROS (fig. S7). Furthermore, treatment with RALF23 suppressed the heightened elf18-induced ROS in s1p-6 mutants (fig. S8), suggesting that the negative regulatory function of S1P is executed by the processing of RALF23 or related peptides. For example, the closely related RALF33 (At4g15800) peptide (fig. S9A, S10A) $(8,9)$ could also inhibit elf18-induced ROS (fig. S6B, S9B,C; S10B). We conclude that RALF23 (and RALF33) negatively regulate immunity.

The Arabidopsis genome encodes about 35 RALF peptides (8, 9). Only 11 RALFs (including RALF23 and RALF33) display a S1P cleavage site (fig. S10A). Co-treatment with RALF34 (containing a predicted S1P cleavage site) inhibited elf18-induced ROS burst to the same extent as RALF23 and RALF33, while RALF32 (lacking a predicted S1P cleavage site) did not (fig. S10B). This suggests that S1P-cleaved RALFs inhibit immunity. RALF23, RALF33, and the more divergent RALF32, triggered seedling growth inhibition (fig. S10C) similar to RALF1 (9). However, RALF32 does not affect elf18-triggered ROS (fig. S10B). All RALF peptides previously tested induced alkalinisation $(8,10)$, suggesting that immune inhibition is not 
a general property of RALFs and is independent of the alkalinisation activity; consistent with our bioassays involving RALF peptides being performed under buffered conditions (Supplement).

The Arabidopsis malectin-like receptor kinase FERONIA (FER; At3g51550) was recently identified as a receptor for RALF1 (9). RALF1, RALF23 and RALF33 are closely related (fig. S9A and S10A), and have overlapping gene expression patterns with FER (fig. S11) $(8,9)$. fer-2 and fer-4 mutants were insensitive to the inhibitory effect of RALF23 or RALF33 peptide on elf18-induced ROS (fig. 2A; fig. S12A), which was complemented in a fer-4/FERGFP line (fig. 2A). Furthermore, RALF23 overexpression in fer-2 had no effect on ROS (fig. S12B). Genetic dependence of FER in RALF23-, RALF33-, and RALF32-triggered growth inhibition (fig. S10C) suggested that FER may bind additional RALF peptides.

Biotinylated RALF23 bound to recombinant FER ectodomain (ectoFER), but not the unrelated EFR receptor ectodomain (ectoEFR) (fig. 2B). Similarly, ectoFER expressed in insect cells bound to biotin-RALF23 but not biotin-elf24 (fig. 2C), with Kd values $\sim 300 \mathrm{nM}$ and $\sim 600$ $900 \mathrm{nM}$ as revealed by microscale thermophoresis and isothermal titration calorimetry, respectively (fig. 2D, fig. S13), consistent with values reported for other ligand-receptor kinase pairs $(11,12)$ and the $\mathrm{IC}_{50}$ for RALF23-mediated inhibition of elf18-triggered ROS (fig. S5). Thus, in addition to RALF1 (9), FER is also a receptor for RALF23.

FER is enriched in detergent-resistant membranes after flg22 treatment (13). The fer-2 and fer-4 mutants were hyposensitive to elf18, flg22, and chitin (fig. 2A, fig. 3A, fig. S12, fig. $\mathrm{S} 14$ ), and were more susceptible to Pto DC3000 COR (fig. 3B), indicating that FER can also positively regulate immunity. FER weakly associates with both FLS2 and BAK1, with the latter being strongly enhanced upon flg22 treatment (fig. 3C). Flg22-induced FLS2-BAK1 complex formation was reduced in fer-4 and restored in fer-4/FER-GFP (fig. 3D). Co-treatment with RALF23 reduced ligand-induced FLS2/EFR-BAK1 complex formation (fig. 3E,F). RALF23 overexpression had a similar effect on flg22-induced FLS2-BAK1 complex formation (fig. S15). RALF23 or the loss of FER did not affect accumulation of FLS2, EFR, or BAK1 (fig. 3D-F, fig. S15). Thus, ligand-induced complex formation between FLS2/EFR and their co-receptor BAK1 is promoted by FER and inhibited by RALF23.

Our data suggest that FER acts as a scaffold to regulate immune receptor complex formation. FER may reside in plasma membrane microdomains as part of pre-formed signaling 'platforms' together with receptors and co-receptors. Whether FER-mediated regulation intersects with other regulators of FLS2/EFR-BAK1 complex formation, such as BIR2 and IOS1 $(14,15)$, will be an interesting topic for future investigation. Two-thirds of RALF proteins lack a predicted S1P-cleavage site and are devoid of a pro-peptide region (fig. S10A). Treatment with one of these, RALF17, induced ROS production and acted additively to elf18 (fig. S16A,B). RALF17 pre-treatment was also sufficient to induce resistance to Pto DC3000 (fig. S16C). As RALF17-induced ROS production is dependent on FER (fig. S16B), the activity is not caused by a possible contamination with synthetic peptides (eg. flg22, elf18) used in our laboratory.

PRORALF23 is processed by S1P within minutes of elicitor perception (fig. S4), suggesting a negative feedback mechanism to inhibit the scaffolding function of FER and dampen immune signaling. Fungal pathogens secrete peptides with homology to RALF23 (16, 17); these fungal RALF orthologs may suppress immunity by inhibiting the formation of active receptor complexes. 
FER has emerged as a regulator of many biological processes ranging from fertilization to inhibition of cell elongation and growth (18). Many of these processes involve receptor kinases and co-receptors (19), suggesting that FER may have a similar scaffolding function within other receptor kinase complexes. Different but overlapping expression patterns of FER and $R A L F$ genes (fig. S11) $(9,20)$ suggest that a variety of FER-RALF modules may regulate diverse receptor kinase complexes during growth, development, or environmental sensing.

\section{References}

1. D. Couto, C. Zipfel, Regulation of pattern recognition receptor signalling in plants. Nat. Rev. Immunol. 16, 537-552 (2016).

2. J. Monaghan et al., The calcium-dependent protein kinase CPK28 buffers plant immunity and regulates BIK1 Turnover. Cell Host Microbe. 16, 605-615 (2014).

3. B. Schwessinger et al., Phosphorylation-dependent differential regulation of plant growth, cell death, and innate immunity by the regulatory receptor-like kinase BAK1. PLoS Genet. 7, 1-17 (2011).

4. C. Rautengarten et al., Inferring hypotheses on functional relationships of genes: Analysis of the Arabidopsis thaliana subtilase gene family. PLoS Comput. Biol. 1, e40 (2005).

5. R. Srivastava, J.-X. Liu, H. Guo, Y. Yin, S. H. Howell, Regulation and processing of a plant peptide hormone, AtRALF23, in Arabidopsis. Plant J. 59, 930-9 (2009).

6. R. Niebergall, Characterisation of potential regulators of PAMP-triggered immunity. https://ueaeprints.uea.ac.uk/42367/ (2012).

7. A. Dobón, J. V. Canet, J. García-andrade, C. Angulo, Novel disease susceptibility factors for fungal necrotrophic pathogens in Arabidopsis, PLoS Pathog. 11, 1-30 (2015).

8. A. Morato do Canto et al., Biological activity of nine recombinant AtRALF peptides: implications for their perception and function in Arabidopsis. Plant Physiol. Biochem. 75, 45-54 (2014).

9. M. Haruta, G. Sabat, K. Stecker, B. B. Minkoff, M. R. Sussman, A peptide hormone and its receptor protein kinase regulate plant cell expansion. Science 343, 408-411 (2014).

10. G. Pearce, Y. Yamaguchi, G. Munske, C. A. Ryan, Structure-activity studies of RALF, Rapid Alkalinization Factor, reveal an essential--YISY--motif. Peptides. 31, 1973-7 (2010).

11. Wang et al., Allosteric receptor activation by the plant peptide hormone phytosulfokine. Nature 525, 265-268 (2015).

12. J. Santiago et al., Mechanistic insight into a peptide hormone signaling complex mediating floral organ abscission. eLife 5, 1-19 (2016).

13. N. F. Keinath et al., PAMP (pathogen-associated molecular pattern)-induced changes in plasma membrane compartmentalization reveal novel components of plant immunity. $J$. Biol. Chem. 285, 39140-9 (2010).

14. T. Halter et al., The leucine-rich repeat receptor kinase BIR2 is a negative regulator of 
BAK1 in plant immunity. Curr. Biol. 24, 134-43 (2013).

15. Y.-H. Yeh et al., The Arabidopsis malectin-like/LRR-RLK IOS1 is critical for BAK1dependent and BAK1-independent pattern-triggered immunity. Plant Cell 7, 1701-1721 (2016).

16. S. Masachis et al., A fungal pathogen secretes plant alkalinizing peptides to increase infection. Nat. Microbiol. 4, 16043 (2016).

17. E. Thynne et al., Fungal phytopathogens encode functional homologues of plant rapid alkalinisation factor (RALF) peptides. Mol. Plant Pathol., doi: 10.1111/mpp.12444 (2016).

18. C. Li, H. Wu, A. Y. Cheung, FERONIA and her pals: functions and mechanisms. Plant Physiol. 171, 2379-2392 (2016).

19. X. Ma, G. Xu, P. He, L. Shan, SERKing coreceptors for receptors. Trends Plant Sci. 21, 1017-33 (2016).

20. E. Murphy, I. De Smet, Understanding the RALF family: a tale of many species. Trends Plant Sci. 19, 1-8 (2014).

21. J.-X. Liu, R. Srivastava, P. Che, S. H. Howell, Salt stress responses in Arabidopsis utilize a signal transduction pathway related to endoplasmic reticulum stress signaling. Plant J. 51, 897-909 (2007).

22. S. D. Deslauriers, P. B. Larsen, FERONIA is a key modulator of brassinosteroid and ethylene responsiveness in Arabidopsis hypocotyls. Mol. Plant. 3, 626-40 (2010).

23. V. Nekrasov et al., Control of the pattern-recognition receptor EFR by an ER protein complex in plant immunity. EMBO J. 28, 3428-38 (2009).

24. Q. Duan, D. Kita, C. Li, A. Y. Cheung, H.-M. Wu, FERONIA receptor-like kinase regulates RHO GTPase signaling of root hair development. Proc. Natl. Acad. Sci. U. S. A. 107, 17821-6 (2010).

25. V. Göhre et al., Plant pattern-recognition receptor FLS2 is directed for degradation by the bacterial ubiquitin ligase AvrPtoB. Curr. Biol. 18, 1824-32 (2008).

26. G. J. Etherington, J. Monaghan, C. Zipfel, D. MacLean, Mapping mutations in plant genomes with the user-friendly web application CandiSNP. Plant Methods. 10, 41 (2014).

27. T. Nakagawa et al., Improved Gateway Binary Vectors: High-performance vectors for creation of fusion constructs in transgenic analysis of plants. Biosci. Biotechnol. Biochem. 71, 2095-2100 (2007).

28. S.-D. Yoo, Y.-H. Cho, J. Sheen, Arabidopsis mesophyll protoplasts: a versatile cell system for transient gene expression analysis. Nat. Protoc. 2, 1565-72 (2007).

29. C. Albrecht et al., Brassinosteroids inhibit pathogen-associated molecular patterntriggered immune signaling independent of the receptor kinase BAK1. Proc. Natl. Acad. Sci. U. S. A. 109, 303-8 (2012).

30. D. Chinchilla et al., A flagellin-induced complex of the receptor FLS2 and BAK1 initiates plant defence. Nature 448, 497-500 (2007) 
Acknowledgments: This research was funded by the Gatsby Charitable Foundation (C.Z.), the European Research Council (grant "PHOSPHinnATE"; C.Z.), the Austrian Academy of Science through the Gregor Mendel Institute (Y.B), the Deutsche Forschungsgemeinschaft (fellowship STE 2448/1 to M.S.), the European Molecular Biology Organization and the United Kingdom Biotechnology and Biological Sciences Research Council (fellowships ALTF 459-2011 and BB/M013499 to J.M), and the Erasmus Mundus program (H.R.). We thank Lena Stransfeld, the JIC horticultural service and the TSL tissue culture service for technical assistance, and all members of the Zipfel laboratory for comments. We thank Nana Keinath, Michael Sussman, Pablo Vera, Sebastian Wolf, Silke Robatzek and Hen-Ming Wu for providing biomaterials, the VBCF Protein Technologies Facility for excellent assistance, and Tim Clausen for access to his ITC platform. M.S., J.M., E.S., Y.B. and C.Z. designed and conceived the experiments, and analyzed the data. M.S., J.M., E.S., H.R., A.L. and Y.B. performed the experiments. N.H. provided unpublished constructs. M.S., J.M., and C.Z. wrote the manuscript with input from all authors. Supplement contains additional data.

\section{Figure legends:}

Fig. 1. The endogenous peptide RALF23 negatively regulates immunity.

(A) ROS production in Col-0 leaf discs treated with $100 \mathrm{nM}$ elf18, $1 \mu \mathrm{M}$ RALF23, or cotreatment (all in $2 \mathrm{mM}$ MES-KOH pH 5.8). Values are means of total photon counts over $30 \mathrm{~min}$ $+/-\mathrm{SE}, \mathrm{n}=16$. Letters indicate significance in one-way ANOVA $(\mathrm{a}-\mathrm{b}, \mathrm{p}<0.001 ; \mathrm{a}-\mathrm{c}, \mathrm{p}<0.001 ; \mathrm{b}-\mathrm{c}$, $\mathrm{p}<0.05$ ). Kinetics are in fig. S18A. (B) Colony forming units (cfu) of Pto DC3000 after syringeinoculation in leaves pre-treated with mock, $1 \mu \mathrm{M}$ elf18, $1 \mu \mathrm{M}$ RALF23, or co-treatment (all in 2 $\mathrm{mM}$ MES-KOH pH 5.8) for $24 \mathrm{~h}$, determined 2 days after inoculation. Values are means +/- SD, $\mathrm{n}=4$ (one-way ANOVA; $\mathrm{p}<0.05$ ). ( $\mathbf{C}$ and $\mathbf{E}$ ) ROS after elicitation with $100 \mathrm{nM}$ elf18 or water. Values are means of total photon counts over $30 \mathrm{~min}+/-$ SE. Letters indicate significance in oneway ANOVA (a-b, p<0.001; a-c, p<0.001; b-c, p<0.05). Kinetics are in fig. S18B,C. (D and F) Number of Pto DC3000 COR ${ }^{-}$bacteria determined 3 days after surface-inoculation. Values are means +/- SD, $\mathrm{n}=4$ (one-way ANOVA; $\mathrm{p}<0.05$ ). Similar results were obtained in 3 independent experiments.

\section{Fig. 2. RALF23-mediated inhibition of immunity is FER-dependent.}

(A) ROS after treatment with $100 \mathrm{nM}$ elf18 alone or co-treated with $1 \mu \mathrm{M}$ RALF23 (all in $2 \mathrm{mM}$ MES-KOH pH 5.8). Values are means of total photon counts over $40 \mathrm{~min}+/-\mathrm{SE}, \mathrm{n}=16$. Letters indicate significance in one-way ANOVA (a-b, p<0.001; $a-c, p<0.05 ; b-c, p<0.01)$. Kinetics are shown in fig. S18D. (B) In vitro binding assay with MBP-ectoFER or MBP-ectoEFR purified from E. coli. Pull-down was done with neutravidin beads; western blots were probed with $\alpha$ MBP. (C) In vitro binding assay with ectoFER-V5-6xHis produced in insect cells. Pull-down was performed with Streptavidin beads; western blots were probed with $\alpha$-V5. (D) Quantitative binding analysis using synthetic RALF23 peptide and ectoFER-V5-6xHis produced from baculovirus-infected insect cells using microscale thermophoresis. Similar results were obtained 
in 3 independent experiments, except for $(\mathbf{C})$ where the assays were performed twice with identical results.

Fig. 3. FER is a RALF-regulated scaffold for immune receptor complexes.

(A) ROS after elicitation with $100 \mathrm{nM}$ elf18 or water. Values are means of total photon counts over $30 \mathrm{~min}+/$ - SE, $\mathrm{n}=8$. Letters indicate significance in one-way ANOVA $(\mathrm{a}-\mathrm{b}, \mathrm{p}<0.05$; $\mathrm{b}-\mathrm{c}$, $\mathrm{p}<0.05 ; \mathrm{b}-\mathrm{d}, \mathrm{p}<0.05 ; \mathrm{a}-\mathrm{c} \mathrm{p}<0.001 ; \mathrm{a}-\mathrm{d}, \mathrm{p}<0.001)$. Kinetics are in fig. S18E. (B) Number of Pto DC3000 COR ${ }^{-}$bacteria 3 days after surface-inoculation. Values are means $+/-\mathrm{SD}, \mathrm{n}=4$ (one-way ANOVA; p <0.05). (C-F) Co-immunoprecipitation of FER-GFP from fer-4/FER-GFP (C), FLS2 from Col-0, fer-4 or fer-4/FER-GFP (D), FLS2-GFP from Col-0/FLS2-GFP (E), or EFR-GFP from $e f r / E F R-G F P(F)$ seedlings treated with or without the indicated peptides $(100 \mathrm{nM} \mathrm{flg} 22$, $100 \mathrm{nM}$ ef118, $1 \mu \mathrm{M}$ RALF23, or water) for $10 \mathrm{~min}$. Western blots were probed with $\alpha$-GFP, $\alpha$ BAK1 and $\alpha$-FLS2. CBB, Coomassie brilliant blue. Similar results were obtained in 3 independent experiments.

\section{Supplementary Materials:}

Materials and Methods

Figures S1-S18

Tables S1-S2

References (21-30) 